\title{
Passeriformes Bird Species as Documented from Conservation Reserve Beed, Jhunjhunu, Rajasthan, India
}

\author{
Vikas Jangir $^{1}$, Meera Srivastava ${ }^{2}$ \\ Department of Zoology, Govt. Dungar College, Bikaner, Rajasthan, India
}

\begin{abstract}
The passerine or the perching birds belong to the largest order of birds Passeriformes, the dominant avian group on Earth today which includes more than half of all bird species and now occur in abundance on all continents except Antarctica and on most oceanic islands. Various habitat characteristics of area viz., topography, vegetation, water availability, nesting sites and other natural as well as manmade structures decide the avian diversity of the region. The present survey was undertaken in the Conservation Reserve Beed of Jhunjhunu district of Rajasthan, India. In all, 65 bird species belonging to 9 families were recorded from the region.
\end{abstract}

\section{Introduction}

The passerine or the perching birds belong to the largest order of birds Passeriformes, the dominant avian group on Earth today which includes more than half of all bird species. These are true perching birds, with four toes, three directed forward and one backward. Considered the most highly evolved of all birds, and now occur in abundance on all continents except Antarctica and on most oceanic islands.

As objects of beauty and interest, passerines have been incorporated into human culture, folklore, poetry, music, sculpture, and painting. Passerines are widely kept as cage birds. Songbirds are economically important in other ways also. Although seldom considered food in economically advanced areas, they are nonetheless important dietary items in many rural or heavily populated countries. Passerines, on the other hand, are serious economic pests. In areas in which one-crop agriculture is extensive, certain bird species have undergone population explosions because of almost unlimited food availability; in turn, their crop depredations can be serious.

As the dominant form of birdlife in virtually all terrestrial environments, the perching birds are a major component of the world's ecosystems. They consume great quantities and varieties of food-grains, fruits, insects and other invertebrates, small amphibians and reptiles, and even small mammals - and in turn serve as food for other animals; they act as hosts for parasites and are occasionally parasitic themselves; they both propagate and distribute plants by pollinating flowers and carrying viable seeds to new locations; and they have the mobility (through migration) to utilize habitats that are available only at certain times of the year.

Various habitat characteristics of area viz., topography, vegetation, water availability, nesting sites and other natural as well as manmade structures decide the avian diversity of the region. The present study was therefore undertaken to document the passerine species status of the recently declared Conservation Reserve at Jhunjhunu district, which would serve as a base line document for future years.

\section{Methodology}

\section{The Study Area}

The present survey was undertaken in the Conservation Reserve Beed of Jhunjhunu district (located between $27^{\circ} 38^{\prime}$ and $28^{\circ} 31^{\prime} \mathrm{N}$ and $75^{\circ} 02^{\prime}$ and $76^{\circ} 06^{\prime} \mathrm{E}$ ) of Rajasthan, India.. The total forest area available is about $10.47 \mathrm{sq} \mathrm{km}$ and is characterized by tropical thorny vegetation represented by Prosopis cineraria, Acacia leucophloea, A. nilotica, Salvadoraoleodes, Capparis decidua, Opuntia sp., Tephrosiapurpuria, Cenchrusciliaris etc.

\section{Materials and Method}

The survey was carried out during a complete annual cycle (2014-15) and the observations were taken on alternate days generally between 5 am to 8 am, sometimes depending upon the season, employing point count method. Olympus binoculars (8x40 DPS I) were used and pertinent field guides (Ali \& Ripley 1983; Grimmett et al. 2001) were followed for identification.

\section{Results and discussion}

The passerine birds documented from the Conservation Reserve Beed of Jhunjhunu during the period of survey have been presented in Table 1. In all, 65 bird species belonging to 9 families were recorded. The family Lanidae dominated the group represented by 14 species, closely followed by Passeridae and Muscicapidae, comprising 13 species each, Silvidae including 9 species, while Stumidae, Hirundinidae, Alaudidae, Pycnonotidae and Nectaninidae were represented by $5,4,4,2$ and 1 species respectively. Further, of the 65 species documented, 41 were resident, 21 were winter migrant, while, summer and passage migrant were represented by two and one species respectively. One very significant point worth mentioning is that, all the documented species are of Least Concerned (LC) category in the IUCN (2012) Red List.Earlier, Sankar et al. (1993), Shahabuddin (2006) and Sultana (2013) have conducted surveys of the Sariska Tiger Reserve of Rajasthan for bird species. 


\section{International Journal of Science and Research (IJSR) \\ ISSN (Online): 2319-7064}

Index Copernicus Value (2013): 6.14 | Impact Factor (2015): 6.391

\section{References}

[1] Ali, S. \& S.D. Ripley (1983). Compact Handbook of the Birds of India and Pakistan. Oxford University Press.

[2] Grimmett, R., C. Inskipp and T. Inskipp (2001). Pocket guide to the birds of Indian subcontinent. Oxford University Press.

[3] IUCN 2012. IUCN Red List of Threatened Species.Version 2012.1.IUCN, Gland, Switzerland. http://www.iucnredlist.org
[4] Sankar, K., D. Mohan \& S. Pandey (1993).Birds of Sariska Tiger Reserve, Rajasthan, India.Forktail,8: 133141.

[5] Shahabuddin, G., R. Kumar \& A. Verma (2006).Annotated checklist of the birds of Sariska Tiger Reserve, Rajasthan, India. Indian Birds, 2 (3): 71-76.

[6] Sultana, Aisha (2013).A checklist of birds of Sariska Tiger Reserve of Rajasthan. Journal of threatened taxa, 5 (13): 4791-4804.

Table 1: Taxonomic list (along with common names) of Passeriformes bird species as documented from Conservation Reserve Beed, Jhunjhunu, Rajasthan, India

\begin{tabular}{|c|c|c|c|c|c|}
\hline \multicolumn{6}{|c|}{ Order: PASSERIFORMES } \\
\hline 1. & \multirow{14}{*}{ Lanidae } & Lanius schach (L.) & Long-Tailed Shrike & $\mathrm{LC}$ & $\mathrm{R}$ \\
\hline 2. & & L. meridionalis (L.) & Southern Grey Shrike & $\mathrm{LC}$ & $\mathrm{R}$ \\
\hline 3. & & L. minor (Gmel.) & Lesser Grey Shrike & $\mathrm{LC}$ & $\mathrm{R}$ \\
\hline 4. & & L. vittatus (Val.) & Bay-Backed Shrike & LC & $\mathrm{R}$ \\
\hline 5. & & Tephrodornis pondicerianus (Gmel.) & Common Woodshrike & LC & $\mathrm{R}$ \\
\hline 6. & & Terpsiphone paradise (L.) & Asian Paradise Flycatcher & LC & $\mathrm{R}$ \\
\hline 7. & & Dendrocitta vagabunda (Lath.) & RufousTreepie & LC & $\mathrm{R}$ \\
\hline 8. & & Corvus splendens (Vieil.) & House Crow & LC & $\mathrm{R}$ \\
\hline 9. & & C. $\operatorname{corax}$ (Wag.) & Jungle Crow & LC & $\mathrm{R}$ \\
\hline 10. & & Rhipidura aureola (Less.) & White-Browed Fantail & $\mathrm{LC}$ & $\mathrm{R}$ \\
\hline 11. & & Dicrurus macrocercus (Vieil.) & Black Drongo & LC & $\mathrm{R}$ \\
\hline 12. & & Aegithina tiphia $(\mathrm{L})$. & Common Iora & $\mathrm{LC}$ & $\mathrm{R}$ \\
\hline 13. & & Oriolus oriolus (L.) & Eurasian Golden Oriole & $\mathrm{LC}$ & SM \\
\hline 14. & & Pericrocotus cinnamomeus $(\mathrm{L})$. & Small Minivet & $\mathrm{LC}$ & $\mathrm{R}$ \\
\hline 15. & & Copsychus saularis $(\mathrm{L})$. & Oriental Magpie Robin & $\mathrm{LC}$ & $\mathrm{R}$ \\
\hline 16. & & Saxicoloides fulicata (Hodg.) & Indian Robin & $\mathrm{LC}$ & $\mathrm{R}$ \\
\hline 17. & & Ficedula parva (Bech.) & Red-Throated Flycatcher & $\mathrm{LC}$ & WM \\
\hline 18. & & Phoenicurus ochruros (Gmel.) & Black Redstart & $\mathrm{LC}$ & WM \\
\hline 19. & & Monticola solitarius $(\mathrm{L})$. & Blue Rockthrush & $\mathrm{LC}$ & WM \\
\hline 20. & & Zoothera wardii (Bly.) & Pied Thrush & $\mathrm{LC}$ & $\mathrm{R}$ \\
\hline 21. & Muscicapidae & Luscinia svecica (L.) & Blue Throat & $\mathrm{LC}$ & WM \\
\hline 22. & & Cercomela fusca (Bly.) & Brown Rock-Chat & LC & $\mathrm{R}$ \\
\hline 23. & & Saxicola caprata (L.) & Pied Bushchat & $\mathrm{LC}$ & WM \\
\hline 24. & & S. torquata $(\mathrm{L})$. & Common Stonechat & $\mathrm{LC}$ & WM \\
\hline 25. & & S. macrorhyncha & Stoliczka'sBushchat & $\mathrm{LC}$ & $\mathrm{R}$ \\
\hline 26. & & Oenanthe picata (Bly.) & Variable Wheatear & $\mathrm{LC}$ & WM \\
\hline 27. & & O. deserti (T.) & Desert Wheatear & LC & WM \\
\hline 28. & & Sturnus pagodarum (Gmel.) & Brahminy Starling & $\mathrm{LC}$ & $\mathrm{R}$ \\
\hline 29. & & S. roseus (L.) & Rosy Starling & $\mathrm{LC}$ & PM \\
\hline 30. & Sturnidae & S. contra (L.) & Asian Pied Starling & $\mathrm{LC}$ & $\mathrm{R}$ \\
\hline 31. & & Acridothere stristis (L.) & Common Myna & $\mathrm{LC}$ & $\mathrm{R}$ \\
\hline 32. & & A. ginginianus (Lath.) & Bank Myna & $\mathrm{LC}$ & $\mathrm{R}$ \\
\hline 33. & & Hirundo rustica (L.) & Barn Swallow & $\mathrm{LC}$ & WM \\
\hline 34. & & H. daurica $(\mathrm{L})$. & Red-Rumped Swallow & $\mathrm{LC}$ & $\mathrm{R}$ \\
\hline 35. & Hirundinidae & H. fluvicola (Bly.) & Streak-Throated Swallow & LC & $\mathrm{R}$ \\
\hline 36. & & H. concolor (Skyes) & Dusky Crag Martin & $\mathrm{LC}$ & $\mathrm{R}$ \\
\hline 37. & & Pycnonotus leucotis (G.) & White-Eared Bulbul & $\mathrm{LC}$ & $\mathrm{R}$ \\
\hline 38. & Pycnonotidae & P. cafer $($ L. $)$ & Red-Vented Bulbul & $\mathrm{LC}$ & $\mathrm{R}$ \\
\hline 39. & & Prinia sylvatica (Jer.) & Jungle Prinia & LC & $\mathrm{R}$ \\
\hline 40. & & P. inornata (Gmel.) & Plain Prinia & LC & $\mathrm{R}$ \\
\hline 41. & & Sylvia curruca (L.) & Lesser White-Throat & LC & WM \\
\hline 42. & & Orthotomus sutorius (Penn.) & Common Tailor Bird & $\mathrm{LC}$ & $\mathrm{R}$ \\
\hline 43. & Silvidae & Phylloscopus humei (Br.) & Hume's Warbler & LC & WM \\
\hline 44. & & P. collybita (Vieil.) & Common Chiffchaff & $\mathrm{LC}$ & WM \\
\hline 45. & & Turdoides caudatus (Dum.) & Common Babbler & LC & $\mathrm{R}$ \\
\hline 46. & & T. malcolmi (Syk.) & Large Grey Babbler & LC & $\mathrm{R}$ \\
\hline 47. & & T. striatus (Dum.) & Jungle Babbler & LC & $\mathrm{R}$ \\
\hline 48. & & Calandrella brachydactyla (Leis.) & Greater Short-Toed Lark & $\mathrm{LC}$ & WM \\
\hline 49. & Alaudidae & Eremopterix grisea & Ashy Crowned Sparrow Lark & LC & $\mathrm{R}$ \\
\hline 50. & Alaudidae & Ammomanes phoenicurus (Frank.) & Rufous-Tailed Lark & $\mathrm{LC}$ & SM \\
\hline 51. & & A. deserti (Lich.) & Desert Lark & LC & WM \\
\hline 52. & Nectarinidae & Nectarinia asiatica (Lath.) & Purple Sunbird & $\mathrm{LC}$ & $\mathrm{R}$ \\
\hline
\end{tabular}


International Journal of Science and Research (IJSR)

ISSN (Online): 2319-7064

Index Copernicus Value (2013): 6.14 | Impact Factor (2015): 6.391

\begin{tabular}{|c|c|c|c|c|c|}
\hline 53. & \multirow{13}{*}{ Passeridae } & Passer domesticus (L.) & House Sparrow & $\mathrm{LC}$ & $\mathrm{R}$ \\
\hline 54. & & P. hispaniolensis (Temm.) & Spanish Sparrow & LC & WM \\
\hline 55. & & Petronia xanthocollis (Bur.) & Chestnut Shouldered Petronia & $\mathrm{LC}$ & $\mathrm{R}$ \\
\hline 56. & & Motacilla alba (L.) & White Wagtail & $\mathrm{LC}$ & WM \\
\hline 57. & & M.maderaspatensis (Gmel.) & White-Browed Wagtail & $\mathrm{LC}$ & $\mathrm{R}$ \\
\hline 58. & & M. flava (L.) & Yellow Wagtail & LC & WM \\
\hline 59. & & M. citreola (Pall.) & Citrine Wagtail & $\mathrm{LC}$ & WM \\
\hline 60. & & Anthus rufulus (Viel.) & Paddy-Field Pipit & $\mathrm{LC}$ & $\mathrm{R}$ \\
\hline 61. & & A. trivialis (L.) & Tree-Pipit & $\mathrm{LC}$ & WM \\
\hline 62. & & A. campestris (L.) & Tawny Pipit & $\mathrm{LC}$ & WM \\
\hline 63. & & A. Hodgi (Rich.) & Olive-Backed Pipit & $\mathrm{LC}$ & WM \\
\hline 64. & & Ploceus philippinus (L.) & Baya Weaver & $\mathrm{LC}$ & $\mathrm{R}$ \\
\hline 65. & & Lonchura malabarica (L.) & Indian Silverbill & $\mathrm{LC}$ & $\mathrm{R}$ \\
\hline
\end{tabular}

$\mathbf{L C}=$ Least Concerned, $\mathbf{R}=$ Resident, $\mathbf{W M}=$ Winter Migrant, $\mathbf{S M}=$ Summer Migrant, $\mathbf{P M}=$ Passage Migrant

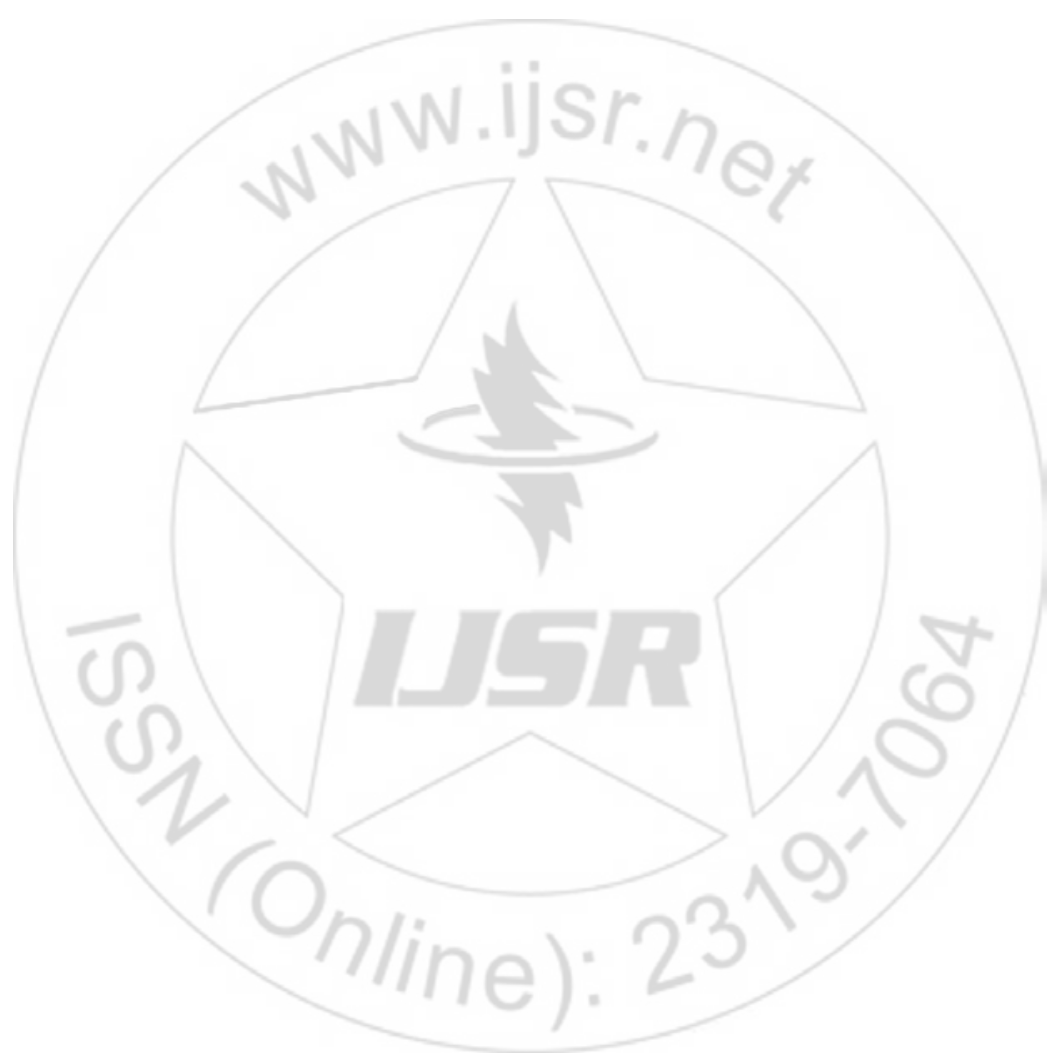

Volume 5 Issue 5, May 2016

www.ijsr.net 